\title{
Analisi sul ruolo degli istituti italiani di cultura e ipotesi di progettazione di nuovi ambienti di apprendimento a rete per la formazione on line dei docenti ${ }^{1}$
}

\author{
Giuseppe Maugeri, Graziano Serragiotto \\ (Università “Ca' Foscari”, Venezia)
}

\begin{abstract}
Questo contributo vuole proporre una riflessione e un'analisi degli Istituti italiani di cultura intesi come agenti di diffusione dello spazio culturale italiano nel mondo. Procedendo dal generale all'analitico, si prenderanno in considerazione gli atti generativi che hanno avviato il MAE all'articolazione di un complesso organizzativo che trova spesso difficoltà di riconoscimento nei vincoli di un apparato considerato troppo rigido e gerarchico nell'attuare delle azioni coordinate e contestuali per lo sviluppo. Successivamente, si definiranno il ruolo e il tipo di contributo che gli Istituti italiani di Cultura (IIC) possono arrecare al Sistema Italia, partendo da una prospettiva di sviluppo e da un'impostazione consapevole di marketing culturale e didattico. Nel far questo, si avanzerà l'ipotesi di un network destinato alla formazione degli insegnanti, le cui implicazioni mostrano come esso possa rendere possibile riconfigurare il sapere e assicurare un'azione IIC più incisiva per migliorare la qualità dei corsi di lingua italiana.
\end{abstract}

Key words: Istituti italiani di cultura. Italiano nel mondo. Difusione della cultura italiana. Formazioni degli insegnanti.

Resumo: Este trabalho tem como objetivo propor uma reflexão e análise dos Institutos Italianos de Cultura entendidos como agentes de difusão do espaço

\footnotetext{
${ }^{1}$ Il contributo è frutto del lavoro congiunto dei due autori che hanno concordato l'impianto generale e la suddivisione in parti. G. Maugeri ha scritto i paragrafi 1-2, mentre G. Serragiotto ha curato il paragrafo 34 e la conclusione (paragrafo 5)
} 
Analisi sul ruolo degli istituti italiani di cultura e ipotesi di progettazione di nuovi ambienti di apprendimento a rete per la formazione on line dei docenti

Giuseppe Maugeri, Graziano Serragiotto

cultural italiano no mundo. Partindo de uma análise geral, serão considerados os atos geradores que associaram o MFA à articulação de um complexo organizativo que muitas vezes torna difícil reconhecer nos vínculos um aparato considerado demasiado rígido e hierárquico para a implementação de ações coordenadas e contextualizadas para o desenvolvimento. Em seguida, serão definidos o papel e o tipo de contribuição que os Institutos Italianos de Cultura (IIC) podem dar ao Sistema Itália, a partir de uma perspectiva de desenvolvimento e do estabelecimento um ambiente consciente de marketing cultural e de ensino. Ao fazer isso, avança-se na hipótese de uma rede para a formação de professores cujas implicações mostram a possibilidade de reconfigurar o conhecimento e assegurar uma ação IIC mais eficaz para melhorar a qualidade dos cursos de língua italiana.

Palavras-chave: Institutos italianos de cultura. Italiano no mundo. Difusão da cultura italiana. Formação de professores.

\section{Il sistema Italia}

In questo paragrafo, si focalizzerà l'attenzione sugli atti costituitivi che hanno condotto a un processo di riforma della Farnesina (2010-2011). A tal riguardo, verranno esaminati dapprima la matrice sui cui è stata impostata una nuova strategia di fondo che completa in modo più profondo quella emanata nel 2000. Nel far questo, si metteranno in evidenza il diverso approccio di sistema, la metodologia dei processi lavorativi e le finalità dichiarate. Successivamente, si dedicherà una riflessione sulle potenzialità del prodotto culturale per il Sistema.

\subsection{Assunti di sviluppo del Sistema}

La configurazione del sistema risponde a un processo di differenziazione con cui si vuole essere percepiti nell'ambiente. Un rapporto sistema-ambiente 
Analisi sul ruolo degli istituti italiani di cultura e ipotesi di progettazione di nuovi ambienti di apprendimento a rete per la formazione on line dei docenti

Giuseppe Maugeri, Graziano Serragiotto

esterno che risulti coerente, non episodico, deve essere costruito sul principio di omogeneità culturale (Weick, 1993), delle competenze interne e di integrazione con il territorio. Un tessuto, dunque, in grado di alimentare una visione, una definizione degli obiettivi e l'implementazione di una strategia di pensiero critica, basata sull'analisi delle situazione delle alleanze (Butera, 1990) e del senso di responsabilità che spinge a identificare ed elaborare delle possibilità di intervento.

Da tali assunti si è sviluppato il progetto di un Sistema MAE che ingloba le migliori qualità dell'Italia per proporsi in uno scenario internazionale con un programma che stimola la credibilità del sistema stesso, il suo riconoscimento come agente di valore, come prodotto/risorsa che, allineato a una logica di mercato, sia interconnesso con i suoi utenti, assicurandone il funzionamento dei servizi erogati e la crescita.

La cultura, in questo caso, è specchio dell'atteggiamento con cui gli elementi comunicano e crescono, dimensionalmente, sul territorio nel quale sono a contatto e per cui lavorano. L'obiettivo comune è costruire una rete che, in termini pragmatici, significa interconnessione, cooperazione come ipoteche per lo sviluppo di nuovi dialoghi, di un confronto con diversi modelli da cui attingere, come strumento di riflessione con cui creare dei legami (Alvesson, 1996). Il sistema è quindi aperto, tende a nuove opportunità, a incrementare se stesso con un livello più alto di conoscenza che va distribuita con trasparenza e continuità.

\subsection{Coordinate di fondo per fare Sistema}

Perché il sistema abbia tutte le componenti utili per proporsi con dei movimenti a caratura internazionale, occorre acquisire alcuni degli elementi riportati sotto che vanno intesi come condizioni necessarie della conoscenza, con relativi ambiti e domini. 
Analisi sul ruolo degli istituti italiani di cultura e ipotesi di progettazione di nuovi ambienti di apprendimento a rete per la formazione on line dei docenti Giuseppe Maugeri, Graziano Serragiotto

1. Conoscenza della struttura, della sua identità:

- saper produrre scambi comunicativi pertinenti al contesto, alla situazione e in rapporto alle competenze richieste a ciascuno, nella specificità dei propri compiti e obiettivi;

- cultura di organizzazione interna che garantisca solidità al sistema e produttività. Fenomeni visibili dell'apprendimento che si misurano sulla base della condivisione di storia e idee, mission e vision chiari e lungimiranti, nonché valori di fondo che a loro volta regolano le connessioni interne e premiano lo sviluppo di contenuti realizzabili e realizzati (Serragiotto, Maugeri, 2012);

- recuperare un grado di coscienza storica che permette a ciascuno di comprendere ed essere parte attiva del cammino dell'organizzazione (Balboni, 2009);

2. Conoscenza delle persone (Williams, Burden, 1997):

- formare ed educare a una dimensione dello sviluppo in cui l'individuo opera con senso del dovere nei confronti dell'organizzazione, esercitando così un ruolo essenziale per il mantenimento della salute del sistema ;

- una grammatica organizzativa, qualunque sia la sua forma, deve prevedere un percorso di sviluppo della persona e di integrazione di idee al fine di moltiplicare le letture della realtà e connettere esperienze di apprendimento differenti (Weick, 1993);

3. Conoscenza della strategia e delle metodologie (Oxford, 1990):

- cogliere nuove prospettive di offerta significa progettare e creare dei processi di collegamento e di interdipendenza con le persone in modo da stabilire una comunicazione e poi un dialogo autentico basato sulla scelta che ciascuno ha di poter dare un contributo. Si tratta di determinare nel personale consapevolezza per l'organizzazione in cui si 
Analisi sul ruolo degli istituti italiani di cultura e ipotesi di progettazione di nuovi ambienti di apprendimento a rete per la formazione on line dei docenti

Giuseppe Maugeri, Graziano Serragiotto

lavora ed essere al tempo stesso guida non statica né direttiva per analizzare e verificare le situazioni;

- dotarsi di strumenti aggiomati ed efficaci (Solima, 2004) che permettano un'analisi del territorio e di intercettare il pubblico potenziale su cui calibrare l'offerta culturale. Un altro fattore importante è che tali metodologie e strumenti devono essere significativi e mirati per avviare un'indagine reale e un focus preciso del territorio;

- strategia di comunicazione (Bollo, 2012) che coordini l'immagine e la diffusione di contenuti all'esterno. In questo caso, vi è rispondenza fra la comunicazione interna ed esterna in quanto la prima è fattore progettuale e costitutivo del secondo;

- una cultura del servizio che produca riflessione, idee, dialogo e abbia come risultato l'aumento della realtà culturale comunicata e prodotta. A tal fine, giocano un ruolo chiave le tecnologie per accelerare e migliorare i flussi di comunicazione interna e raggiungere un'utenza ad hoc.

L'approccio caratterizzante ripone nell'interdipendenza delle persone e nella loro cultura organizzativa il senso della crescita del sistema che va ridefinito come continuum di cambiamento dei singoli nella forma di una comunità di appartenenza che, agendo in un contesto sociale ed affettivo, si riposiziona come modello di una cultura che esprime e definisce in sé e nel dialogo con l'ambiente il senso della propria identità e del ruolo di responsabilità che diventa espressione irrinunciabile della propria missione. Ecco perciò che si viene delineando la smaterializzazione di un prodotto che ha la forma della conoscenza che si diffonde fra le persone e viene socialmente condivisa in sistema di appartenenza. Partendo da questa ipotesi di fare sistema, si può parlare di un diverso orientamento nel quale è la stessa organizzazione a esprimere la cultura nazionale e articolarla in forme ibride di competenze e apprendimento. 
Analisi sul ruolo degli istituti italiani di cultura e ipotesi di progettazione di nuovi ambienti di apprendimento a rete per la formazione on line dei docenti Giuseppe Maugeri, Graziano Serragiotto

\subsection{Analisi organizzativa del sistema MAE}

Il Sistema così come è stato concepito, prende le mosse dalla volontà strategica di:

a) adeguarsi alle direttive di fondo che connaturano il vigente Sistema europeo per l'azione esterna;

b) gestire e coordinare la complessità.

Quest'ultimo fattore rappresenta un'opportunità perché può portare l’organizzazione a differenziare i bisogni e le opportunità per lo sviluppo. In questo caso, il Sistema ha avuto la necessità di semplificare le strutture portanti e costituire una rete in modo da allargare l'arena decisionale a diverse unità interrelate fra di loro e avere, al contempo, diversi orizzonti impegnati ad attuare la riflessione strategica sulle priorità e gli indirizzi da seguire, sulla gestione e sulla produttività dell'intero organismo. Il rischio, come si è già constatato, è di sovrapporre i livelli decisionali e allungare la catena di comando.

La natura stessa del sistema, inoltre, ci informa sull'evoluzione di un progetto vivente che vuole rapportarsi con il contesto locale e internazionale che lo avvolge (Caon, Maraschio, 2011). Si tratta di un ambiente che stabilisce sempre nuovi confini, in cui si individuano strutture di pensiero, gruppi e comunità che muovono all'azione e spingono alla costruzione di significati con lo scopo di generare miglioramenti della qualità della vita dei partecipanti. In questo senso, il ruolo delle sedi estere ha acquisito una maggiore rilevanza grazie a un coordinamento più efficiente e una maggiore razionalizzazione della gestione complessiva di tali organismi e delle risorse umane che operano all'estero.

Da questa prospettiva, si configura un sistema MAE razionale e ordinato, che definisce la sua natura biologica e il suo ruolo ben preciso attraverso interventi 
Analisi sul ruolo degli istituti italiani di cultura e ipotesi di progettazione di nuovi ambienti di apprendimento a rete per la formazione on line dei docenti Giuseppe Maugeri, Graziano Serragiotto

tematici che abbracciano le diverse aree geografiche in cui si situa l'intervento dell'Italia. Il livello di organizzazione presenta una ramificazione interna che consente alle singole Direzioni (per un totale di otto) che lo compongono di affrontare le sfide a livello nazionale/globali e di avanzare delle ipotesi e strategie di lavoro sinergiche e possibili. Una struttura, dunque, che possiede un equilibrio interno dove ogni Direzione ha una sua collocazione e funzionalità chiara.

In questo scenario, la cultura è componente attiva del processo di costruzione delle identità, di formazione e di comunicazione di un sistema coordinato da un livello gerarchico riconoscibile nel modo di guardare al futuro e di contribuire in modo imprenditoriale allo sviluppo di un'economia della conoscenza. A tal fine, si deve distinguere fra cultura interna e un'altra orientata al mercato. Per quanto riguarda alla prima, sarebbe auspicabile accertarsi del livello di cultura interna al MAE, indagando i livelli di appartenenza, riconoscimento dei dipendenti alla struttura e definire in questo modo il quadro delle regole, dei principi condivisi e dei simboli che, come detto, rientrano a pieno titolo nella dimensione valoriale dell'organismo.

In riferimento al contributo per la cultura da destinare alla sfera esterna, invece, il MAE presenta una propria unità lavorativa con la Direzione Generale per la promozione del Sistema Paese. Essa si pone l'obiettivo di individuare in maniera prospettica le potenzialità che un determinato contesto suggerisce in termini di interesse e di destinazione più adatta del prodotto culturale italiano. La sua articolazione in otto sezioni o uffici, colloca la gestione degli IIC al IV ufficio che pertanto si occupa di attenzionare $i$ processi di sviluppo degli IIC così come la gestione delle risorse da erogare a tali istituzioni. Compito delicato e non meno importante visti i tagli alla cultura. 
Analisi sul ruolo degli istituti italiani di cultura e ipotesi di progettazione di nuovi ambienti di apprendimento a rete per la formazione on line dei docenti Giuseppe Maugeri, Graziano Serragiotto

In sintesi, si vuole riportare una scheda che riassuma le principali istanze di rinnovamento adottate dal MAE a seguito dell'evoluzione del contesto europeo e di una azione diplomatica differente introdotta con il D.P.R. del 19 maggio 2010.

\section{Contesto europeo}

Adeguandosi strutturalmente ai principali partner europei che in questi anni si sono resi protagonisti di aggiustamenti di piccole o grandi dimensioni nell'organismo dei ministeri degli Esteri e nella loro rete diplomatica .

In vista della partecipazione al Servizio Europeo d'Azione Esterna in vigore col Trattato di Lisbona che costituisce il primo nucleo di una azione diplomatica a livello europeo, la nuova struttura del MAE italiano vuole rispondere in modo proattivo e dinamico alle sfide e aspettative future dell'Europa, con particolare riguardo alla sicurezza e globalizzazione.

Novità introdotte dal D.P.R. 19 maggio 2010

Frutto di una valutazione comparate delle iniziative dei principali Paesi partner, le cifre distintive che caratterizzano il piano di rinnovamento strutturale del MAE mirano a comunicare rispettivamente:

a) un senso profondo del ruolo che l'Italia si pone di svolgere nello scenario internazionale;

b) centralità dell'approccio tematico al fine di assicurare un efficace e continuo processo di coordinamento.

Da un punto di vista strutturale, le novità più evidenti consistono:

a) nella riduzione delle Direzioni Generali 13 a 8 allo scopo di razionalizzare le risorse umane e semplificare l'apparato; 
b) nella ridefinizione delle competenze organizzate secondo una priorità d'azione tematica e non più geografica;

c) Affari politici e sicurezza, mondializzazione e questioni globali, integrazione europea, promozione del sistema paese.

La parola chiave è coordinamento e coerenza: a questo compito sono preposte due figure quali il SG e il Consiglio di Amministrazione che esprime valutazione sugli indirizzi strategici e sull'azione complessiva del Ministero.

Lingua e cultura italiana: la Direzione Generale per la Promozione del Sistema Paese

Le attività di sostegno e di proiezione internazionale in campo culturale fanno capo adesso al DG per la Promozione del Sistema Paese. In questa sede, vengono esplicitati gli assunti di base del nuovo assetto che poggia principalmente sulla complementarietà fra cultura ed economia, individuando come strategia per lo sviluppo complessivo un approccio di sistema e le risorse intellettuali in qualità di componenti per rafforzare la promozione della lingua e della cultura italiana nel mondo. In modo specifico, la DGSP si pone l'obiettivo di:

a) gestire gli IIC e la rete di scuole operanti all'estero;

b) progettare, programmare e far circuitare gli eventi culturali grazie alla distruzione capillare delle sedi istituzionali estere;

c) accelerare il processo di internazionalizzazione degli atenei italiani.

\subsection{La dimensione produttiva della cultura}


Analisi sul ruolo degli istituti italiani di cultura e ipotesi di progettazione di nuovi ambienti di apprendimento a rete per la formazione on line dei docenti Giuseppe Maugeri, Graziano Serragiotto

L'attuale frangente politico-economico impone una revisione delle strategie e, di conseguenza, delle strutture predisposte per conseguire delle azioni efficaci di politica estera. L'evoluzione dello scenario internazionale da un punto di visto geopolitico ha di fatto comportato un differente assetto degli equilibri internazionali per cui è lo Stato ad assumersi la responsabilità di dirigere il mercato e garantire lo sviluppo. Obiettivo che può essere conseguito con una gestione aziendale della cosa pubblica, secondo una logica della progettazione e della gestione che si richiama ad assunti basati sulla razionalizzazione e ottimizzazione delle risorse, sull'aumento della competitività e della produttività e di una valutazione non solo legata ai risultati conseguiti, ma anche del processo al fine di analizzare ed elaborare tutti i momenti dell'acquisizione (Serragiotto, 2006). Il suddetto quadro di riferimento, trova un suo momento di produttività nell'investimento in cultura.

Per prima cosa, la cultura che rappresenta lo strumento per indirizzare l'organizzazione verso l'operatività di modelli manageriali efficaci. Aumentando il nesso fra persone e organizzazioni, la cultura giustifica i processi decisionali, la resa più estesa della performance del soggetto che cresce in forza del problem solving e delle prospettive di comprensione e di lettura della realtà. L'utilizzo strumentale della cultura, inoltre, si discosta dall'atteggiamento meccanicistico e ininfluente con cui essa è stata finora considerata; l'importanza che essa assume all'interno di un sistema che promuove se stessa fa luce sugli elementi di qualità interna che le persone che lo compongono devono avere in termini di formazione e continuo aggiornamento della propria professionalità sincronizzata sulla appropriatezza della cultura organizzativa messa in atto. È evidente allora come il livello di esistenza e di significato assunti dal Sistema è strettamente connesso con la cultura organizzativa e l'operatività con cui essa trasforma modelli concettuali 
Analisi sul ruolo degli istituti italiani di cultura e ipotesi di progettazione di nuovi ambienti di apprendimento a rete per la formazione on line dei docenti Giuseppe Maugeri, Graziano Serragiotto

e organizzativi in azioni precipue, tattiche e strategiche, finalizzate. Questa diversa consapevolezza circa il sistema valoriale da cui attingere dalla cultura, fa sì che si crei un pensiero che guidi il processo di creazione e di intervento appropriato nei contesti. In questa direzione, la promozione della cultura italiana deve essere frutto di un'attenta progettazione e programmazione di eventi e attività finalizzati ai seguenti aspetti:

a) sostenere la domanda di italiano; si tratta di un elemento che presuppone un'analisi e conoscenza approfondita del territorio in cui si opera;

b) creare delle situazioni associate al consumo del bene intangibile (Fabris, 2003): più che proporre degli eventi, bisogna progettare dei momenti di partecipazione misurati sulla base dei bisogni e delle motivazione degli utenti, nonché sul livello di soddisfazione assegnato all'evento stesso;

c) stabilire un dialogo differenziato sul prodotto linguistico e culturale: anche in questo caso, la priorità è rivolta alla comunicazione con l'utente come mezzo per conoscere le sue richieste/ aspettative, e rendere accessibile il bene;

d) determinare un processo esperienziale di apprendimento che permetta un livello profondo di partecipazione e di riconoscimento della formazione maturata presso la comunità culturale.

Nel caso della lingua e della cultura italiana, si tratta di costruire un approccio di sistema per mezzo di cui fornire delle risposte pertinenti al proprio pubblico (docenti e studenti, cultori dell'italianità nell'espressione differenziata e ricca delle sue forme). In questo modo, gli organismi competenti per la diffusione della lingua italiana potranno esprimere aspetti di sé e veicolare in modo sistematico una relazione significativa per la valenza culturale e critica dell'esperienza prodotta di cui essi sono espressione. 
Analisi sul ruolo degli istituti italiani di cultura e ipotesi di progettazione di nuovi ambienti di apprendimento a rete per la formazione on line dei docenti Giuseppe Maugeri, Graziano Serragiotto

\section{Gli Istituti italiani di cultura}

L'analisi pragmatica con la quale si intende cogliere questo ambito di studio e di ricerca è quello di considerare il settore indagato come campo sociale interrelato con altri spazi conoscitivi quali la glottodidattica, le neuroscienze e l'analisi contestuale del marketing culturale. Del resto, gli attori che si identificano sono responsabili di obiettivi sociali ed educativi la cui mediazione con la realtà produce un fenomeno eterogeneo su cui occorre impostare e riconoscere una teoria di progetto e una precisa metodologia di azione.

Dunque, nei prossimi paragrafi ci si propone di qualificare i recenti studi di promozione della lingua italiana all'estero che si stanno imponendo al fine di descrivere i riferimenti scientifici e culturali, logici e tecnici la cui valenza dispiega uno spazio progettuale che spinge gli IIC alla costruzione di eventi e alla chiarificazione di effetti di senso a ogni atto comunicativo. Nello specifico della didattica dellitaliano, si proporrà poi un'ipotesi progettuale di formazione dei docenti a rete mediata attraverso l'uso delle moderne tecnologie. L'obiettivo è di costituire una prima azione di sviluppo e di riconoscimento dei docenti IIC come comunità di pratica che attualizza il sapere sulla base di una preparazione glottodidattica e di un livello di attenzione maggiore del contesto in cui si opera, partendo proprio dall'utilizzo della tecnologia per sottolineare il valore aggiunto che tali strumenti consentono nell'ottica di un apprendimento fruibile in tutti i luoghi in cui le persone si trovano.

\subsection{Il ruolo degli Istituti Italiani di Cultura nel Sistema}

Estranea alla cultura imprenditoriale e borghese, la spinta individualistica e interventista in materia di politica linguistica e culturale, ha spinto lo Stato italiano ad assumere diversi ruoli di partecipazione e mediazione, spesso senza 
Analisi sul ruolo degli istituti italiani di cultura e ipotesi di progettazione di nuovi ambienti di apprendimento a rete per la formazione on line dei docenti

Giuseppe Maugeri, Graziano Serragiotto

peso, erogando finanziamenti e somme tali con lo scopo di dare impulso al prodotto italiano. Rileggendo Cummins, Severino (2012) sostiene che nel periodo di transizione economica che si antepone all'attuale recessione economica internazionale, ovvero dagli anni 70 ai 90 , il funzionamento dell'organizzazione ministeriale preposta alla diffusione del Made in Italy è avvenuto senza un coordinamento di ciò con il prodotto era avvolto e in cui era immerso: la cultura italiana. L'involucro culturale espressione tangibile e visibile dei prodotti italiani è cresciuto dietro la spinta di azioni locali che non rientravano in una progettualità ad hoc. Erano semmai delle singole, isolate e contestuali reazioni a forme di interesse dei meccanismi dell'italianità. Curiosando nelle statistiche riportate da Giovanardi e Trifone (2012), è evidente che il fenomeno della lingua italiana cresca nella misura in cui veniva proposta in modo univoco e coerente delle offerte di apprendimento esperienziali da parte dell'IIC. Senza scendere nei particolari di ogni azione promozionale, una lettura fra le righe e più approfondita permette di cogliere alcuni aspetti significativi in relazione ai comportamenti tenuti dall'organizzazione culturale in Giappone in occasione della promozione dei corsi. In esse si riconoscono delle leve strategiche del marketing didattico, quali:

a) la ridefinizione della strategia culturale; essa viene configurata sulla base della missione e degli obiettivi dell'organismo, da cui scaturiscono delle ricerche mirate a individuare le specifiche dei potenziali clienti;

b) un rafforzamento della posizione IIC sul mercato delle lingue straniere; da questa fase si ottiene sia una segmentazione del mercato che un posizionamento in esso. $\mathrm{Si}$ creano pertanto le basi per stabilire una matrice delle relazioni con il target individuato (Franceschini, 1998);

c) l'obiettivo di apprendimento cardine della relazione con lo studente (Boaretto et al., 2011): riprendendo il punto $b$, in questa fase ci si 
Analisi sul ruolo degli istituti italiani di cultura e ipotesi di progettazione di nuovi ambienti di apprendimento a rete per la formazione on line dei docenti Giuseppe Maugeri, Graziano Serragiotto

focalizza sulla volontà di creare dei legami forti con lo studente in tutti i punti in cui avviene il contatto fra le due parti. A questo scopo, gli strumenti multicanali potrebbero allargare le possibilità di accesso alle informazioni ed elevare l'interattività coordinandola sempre con le finalità che si acquisiscono aderendo ai corsi di lingua italiana;

d) il livello e l'intensità di comunicazione; riconsiderando gli enunciati del punto $c$ e le condizioni infrastrutturali del macroambiente, la convergenza multimediale della comunicazione offre la possibilità di aumentare la frequentazione all'impresa anche sotto forma di interazione on line, di disponibilità di contenuti, di dialogo e di negoziazione, di integrazione di contributi e di richieste specifiche dello studente;

e) la centralità del cliente/studente nel processo di co-costruzione delle sue prospettive future. In una condizione di apprendimento organizzato, vi è l'urgenza di mantenere lo studente al centro del processo di costruzione della conoscenza. Nell'ambito di una progettualità orientata all'allievo, quest'ultimo svolge un ruolo attivo nel definire il significato di ciò che studia.

L'ipotesi plausibile che giustifica il successo delle iscrizione presso numerosi sedi estere si riconduce, in linea generale e dalle conversazioni avute con $i$ responsabili didattici, al un funzionamento organizzativo, a una progettazione e una pianificazione di interventi di natura intellettuale distribuiti organicamente nel territorio. Inoltre, il sistema comunicativo è riuscito a intercettare e legare il target mediante delle forme discorsive efficienti. L'uso di un linguaggio contestuale e interculturale, ampio ed efficace, rimanda a una riflessione sul contesto e sulle strategie di azione allo scopo di calare una prospettiva di apprendimento contestuale all'ecologia dell'ambiente e ai bisogni delle diverse categorie di studenti. In tal modo, le offerte proposte si 
Analisi sul ruolo degli istituti italiani di cultura e ipotesi di progettazione di nuovi ambienti di apprendimento a rete per la formazione on line dei docenti Giuseppe Maugeri, Graziano Serragiotto

differenziavano per contenuti e linguaggi, risorse ed eventi comunicativi distinti e distintivi di un accrescimento della collettività di apprendenti .

Attuare in modo sistematico e situato una strategia paese, significa ascoltare in modo attivo le richieste formative che giungono dal contesto, leggere i diversi scenari in cui si opera e coordinare delle proposte progettuali da presentare in un dato segmento di mercato secondo una direzione ben definita nella metodologia come nei risultati auspicati dagli attori.

Il movimento culturale di un tale soggetto istituzionale dello spessore degli IIC si misura dunque anche dalla capacità di sviluppare degli strumenti di crescita della comunità culturale (Serragiotto, Maugeri, 2012), realizzando e gestendo i corsi di lingua italiana. Si tratta di offerte culturali che mirano a implementare delle azioni rivolte al target effettivo e potenziale, a incrementare la partecipazione e il contatto con i nuovi consumatori, a creare un dialogo aperto che sia fonte di valore e di interessi per entrambi. Ma qualsiasi architettura dei corsi di italiano promossi, deve necessariamente poggiare sul pilastro della qualità che costituisce l'oggetto di analisi nel prossimo paragrafo.

\subsection{Aspetti identificativi degli IIC: la qualità}

Per prima cosa, la qualità si riferisce a tutti gli aspetti della vita dell'ente erogatore di cultura e a tutte le forme di significato espresse in un dato contesto. In parole concrete, la qualità si applica a tutti gli aspetti della vita dell'organismo. Questo presuppone come tale fattore abbia come condizione uno spazio di consumo didattico allargato a ogni ambiente dell'istituto in cui la conoscenza fluisce in modo circolare a tutti gli attori (Butera, 1990). Si tratta quindi di concepire la qualità secondo un'idea sferica diffusa a tutti i livelli di creazione dell'apprendimento. Di conseguenza, l'esperienza olistica generata, riguarda ogni interfaccia dell'ambiente di studio. Ne scaturiscono dei livelli di 
Analisi sul ruolo degli istituti italiani di cultura e ipotesi di progettazione di nuovi ambienti di apprendimento a rete per la formazione on line dei docenti

Giuseppe Maugeri, Graziano Serragiotto

comunicazione e interazioni diversi che fanno leva sulla costruzione delle competenze e di processazione di tutte quelle informazioni necessarie ad arrecare un valore aggiunto all'esperienza di studio degli allievi.

Nella prospettiva di analisi adottata, i costrutti della qualità sono olistici e soggettivi (Hofstede et al. 1990, Alvesson, 1996), analitici e fondati sul rapporto emozionalità-razionalità degli individui. In ogni caso, essa è compresa nell'esperienza che compiono i soggetti a partire dai punti di contatto che hanno avuto con l'organizzazione. Una di queste, potrà essere la sede che si valuterà rispettivamente nella sua componente hard e soft.

a) la sede intesa come fatto sociale esterno (Durkheim, 1996); tale struttura riveste un ruolo altamente strategico poiché attrae, permette di essere riconosciuta, avvicina per l'esclusività degli elementi architettonici e raccoglie per l'identità che è capace di comunicare. In questa prospettiva, la struttura possiede una propria concezione del sapere e una individualità relazionale con cui si propone e si definisce visivamente nel territorio. Ne consegue che il territorio si determina come scenario ideale per costruire storicamente il luogo e consolidare una strategia di carattere politico-economica e sociale coordinata, specifica e situata (Lave, Wenger, 1991). Per azioni situate, perciò, si intendono degli eventi, proposte formative e culturali che presentano una rete di significati reali e comprensibili nel-dal contesto (Ricoer, 1978);

b) sede come fatto sociale interno: con la seguente definizione si fa riferimento alla sedimentazione dei fenomeni storico-culturali che hanno prodotto la portata reale della sua incidenza nell'ambiente (Balboni, 2006). Con tale definizione, ci si riferisce a una sede che è sfondo dell'azione sociopolitica e culturale dell'ente operante in un contesto estero presso cui ha messo a disposizione delle risorse, 
Analisi sul ruolo degli istituti italiani di cultura e ipotesi di progettazione di nuovi ambienti di apprendimento a rete per la formazione on line dei docenti

Giuseppe Maugeri, Graziano Serragiotto

partecipando così alla costruzione della conoscenza. Dunque, dall'analisi di come l'IIC si presenta si possono ricostruire il tipo di strategia messa in atto e i livelli di interazione che si sono svolti nell'arco di periodi storici caratterizzanti la propria presenza in loco.

La ricerca storica e documentaristica testimonia come le trasformazioni che le sedi hanno dovuto subire in molte circostanze non risultano essere allineati con i principi di sviluppo per Paese delineati all'origine a livello macro. Basti pensare a un modello di Istituto che segue una modalità di avvicinamento al territorio definita come "sportello unico". In casi come questi, gli spazi culturali della sede culturale, sono stati interamente assorbiti dall'ambasciata, regolando le componenti soft a vincoli in parte estranei alle prospettive di interazione IIC col suo pubblico, rendendo di fatto più complesso l'accesso degli utenti presso la sede in quanto soggetta a maggiori controlli. Gli assunti di visibilità e di riconoscimento sociale risultavano così legati a una situazione che aveva ridotto i processi sociali di partecipazione della sede, di relazione con il pubblico, di utilizzo in loco dei materiali.

Sulla base dei punti indicati, l'influenza dei fattori strutturali esterni si proietta sui vincoli degli spazi di lavoro interni, le cui componenti soft (regole, norme, cultura, organizzazione degli spazi, ecc.) descrivono a livello micro ambientale la coerenza fra il livello di comunicabilità esterna e quella interna (Distratis et al., 2011). Al susseguirsi quindi della personalità dell'Istituto (strutturale esterna, ambienti di lavoro interni) si aggiunge una reciprocità (Giddens, 1991) fra organizzazione degli ambienti e azioni conoscitive a scopo didattico e di apprendimento olistico indirizzate al gruppo di individui che partecipano al processo di utilizzo degli spazi, delle tecnologie di cui si dispone, del prodotto linguistico (fig. 1). 
Analisi sul ruolo degli istituti italiani di cultura e ipotesi di progettazione di nuovi ambienti di apprendimento a rete per la formazione on line dei docenti Giuseppe Maugeri, Graziano Serragiotto

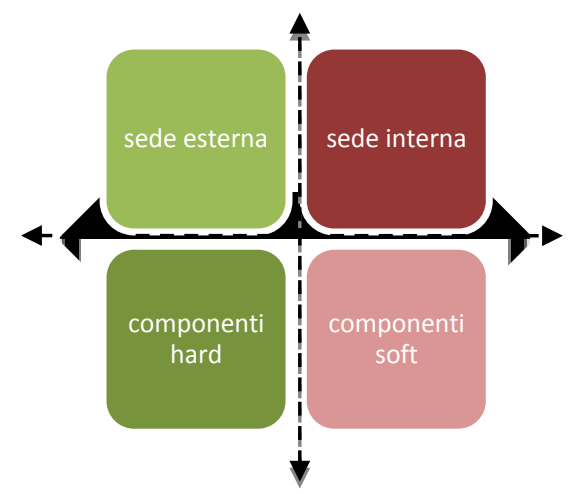

Fig. 1. Interfacce della realtà progettuale degli spaz̧i di apprendimento. Nostra elaborazione.

\subsection{La qualità nell'organizzazione}

Considerando tale paradigma di rielaborazione della conoscenza, la qualità va identifica in tutti gli aspetti della scuola, e nello specifico, si realizza in una struttura organizzativa coesa e capace di assicurare-accelerare un ritmo di apprendimento personalizzato sulla base di azioni congiunte, coordinate e condivise fra i diversi attori con lo scopo di promuovere un sistema di studio e di acquisizione della lingua con lo scopo di:
a) favorire forme l'integrazione;
b) coordinare il processo di apprendimento;
c) gestire in modo proattivo le criticità;
d) intervenire con soluzioni personalizzate;
e) assicurare il coinvolgimento e la partecipazione di tutti;
f) adottare strumenti di insegnamento specifici;
g) formare ad apprendere mediante didattiche esperienziali;
h) sostenere e creare collegamenti, scambi e azioni finalizzati a rendere effettivo spendibile l'acquisizione.

Dunque, la qualità si manifesta in modo visibile e latente in ogni ambiente dell'istituto che si rivela propizio per innescare l'apprendimento dello 
Analisi sul ruolo degli istituti italiani di cultura e ipotesi di progettazione di nuovi ambienti di apprendimento a rete per la formazione on line dei docenti

Giuseppe Maugeri, Graziano Serragiotto

studente. La qualità di ogni livello contestuale e didattico agevola il contatto fra l'istituto e lo studente, migliorando l'aspetto relazionale e comunicativo di entrambi, i processi decisionali e di comprensione del fenomeno linguistico (fig. 2).

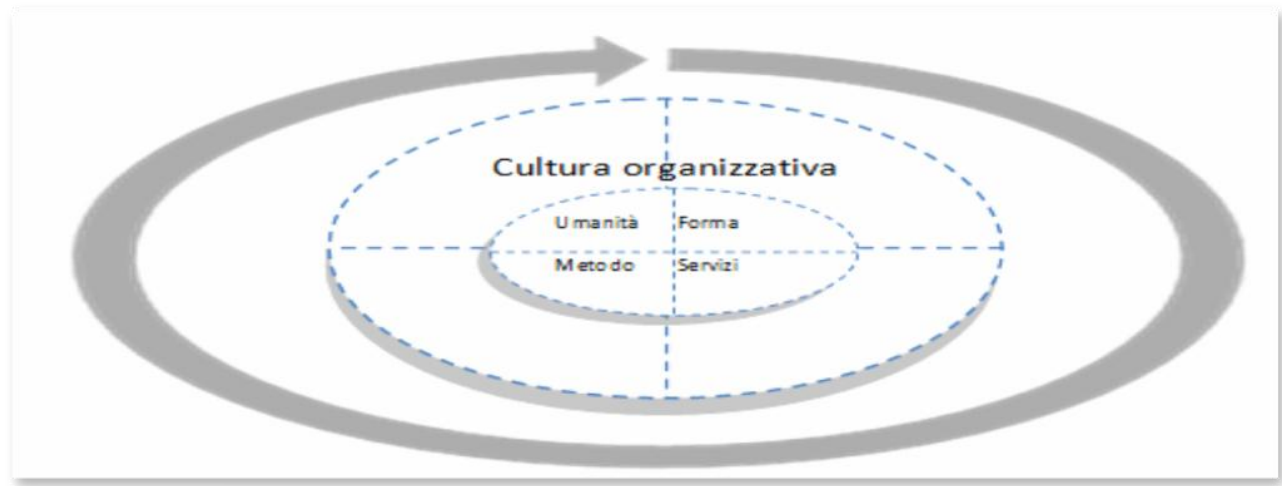

Fig. 2. Architettura simbolica dell'organizzazione culturale. In Serragiotto, Mangeri, 2012.

Da questa prospettiva, la qualità ha una valenza sociale dal momento che può essere misurata in base al livello di socializzazione raggiunta, nonché grazie agli strumenti che misurano la soddisfazione che lo studente ha avuto nei confronti dell'esperienza di studio globale, dal generale al particolare.

\subsection{La qualità nella formazione delle competenze delle persone}

Considerando gli elementi costitutivi che connaturano una sede istituzionale come gli IIC, aspetti quali creatività e rigore devono necessariamente convivere in una visione d'impresa produttiva, rappresentando un nodo fondamentale dei processi di valorizzazione delle persone e delle connessioni che strutturano il sistema reticolare della conoscenza. In quest'ottica, vengono riconosciuti i legami instaurati dagli individui che diventano pertanto soggetti di una comunicazione aperta, di pratiche operative e di lavoro flessibili e 
Analisi sul ruolo degli istituti italiani di cultura e ipotesi di progettazione di nuovi ambienti di apprendimento a rete per la formazione on line dei docenti Giuseppe Maugeri, Graziano Serragiotto

cooperative. Meccanismi di coesione e di regolamento del sistema come la socialità e le connessioni, quindi, trovano espressione nella concezione umanistica dell'organismo che fonda le sue proprietà sulla comunità delle persone, sulla reciprocità e sulle interdipendenze fra le diverse aree.

In questo senso, vanno integrati nella progettazione dei percorsi finalizzati allo sviluppo delle competenze delle persone, dal momento che tali momenti costituiscono la vitalità del sistema stesso. Un'impresa che si focalizza sulle competenze degli individui determina delle forme di continuità e di evoluzione fra la dimensione culturale esterna e interna, operando così in sinergia con il contesto. Secondo questa modalità, i confini fra la dimensione interna e quella esterna diventano labili per il fatto che non si agisce mai fuori da un contesto specifico. L'ambiente esterno dà sostegno in qualche modo all'ambiente interno (Hofstede, 1985) per cui nel primo si possono riconoscere $\mathrm{i}$ tratti specifici di quelli esterno e viceversa. Da questa prospettiva, l'impresa si configura come un sistema aperto e interconnesso così come lo sono le persone che realizzano processi compatibili con la realtà esterna.

Procedendo da questo punto di vista, la comunità delle persone diventa il fattore strategico dell'organizzazione poiché l'individuo è considerato un soggetto portatore di idee e di cultura e pertanto sarà egli stesso a determinare il senso della formazione sulla base delle proprie priorità e orientamenti pratici e affettivi.

Su questa linea, la formazione assume un significatività maggiore in quanto un luogo che permette rispettivamente di:

a) essere consapevoli di un modello culturale unitario che ispira; 
Analisi sul ruolo degli istituti italiani di cultura e ipotesi di progettazione di nuovi ambienti di apprendimento a rete per la formazione on line dei docenti Giuseppe Maugeri, Graziano Serragiotto

b) comprendere le differenze culturali tra le persone; ciò significa creare la disposizione negli individui di saper ascoltare e agire all'interno di un gruppo (Sulkunen, 1982) che impara ad imparare (Serragiotto, 2004);

c) essere informati sulla missione di fondo;

d) offrire dei criteri e degli strumenti intellettuali e di riflessione critica aperta nell' interpretare i cambiamenti (Ouchi, Wilkins, 1985);

e) avere un modo comune di risolvere i problemi (Pfeffer, 1994);

f) sapere realizzare i processi secondo un fine comune;

g) avere una cultura ad alto grado di competenza e produttività;

h) fondare i costrutti teorici e i modelli pratici per conferire un significato profondo alla propria autonomia.

In questa accezione, le persone rappresentano la metafora dell'organizzazione che si relazione con la realtà e nello stesso tempo apprende il modo di porsi in linea con la cultura interna e con la natura culturale del contesto. Questa prospettiva di "personalizzare l'organizzazione e precisare il suo linguaggio sulla base di esigenze ambientali", gioca sull'autonomia, la capacità di problem solving (Levy, 1996), sulle relazioni e competenze degli individui nel tradurre il sapere in quadri di riferimento teorico e pratiche di lavoro efficienti. In questo modo, si colgono le implicazioni profonde dell'organismo e lo si sostengono attraverso la conoscenza tacita (Nonaka, Takeushi, 1995) e condivisa del capitale intellettuale e la specificità cognitiva richiesta a ciascuno.

\subsection{La qualità del prodotto linguistico}

La qualità del processo di organizzazione che, come si è constatato, coinvolge tutte le sfere dell'sstituzione, riguarda in misura evidente il processo di costruzione della conoscenza, per cui essa si estende anche sul livello qualitativo del prodotto intangibile. Dunque, i fondamenti della qualità 
Analisi sul ruolo degli istituti italiani di cultura e ipotesi di progettazione di nuovi ambienti di apprendimento a rete per la formazione on line dei docenti Giuseppe Maugeri, Graziano Serragiotto

dell'organizzazione diventano visibili nelle politiche linguistiche attuate, spostando i loro significati nelle offerte culturali messe in campo come nel caso dei corsi di italiano che acquistano profondità se rivelano il nesso fra cultura dell'organizzazione e la qualità metodologica didattica dei corsi. In questo senso, un modello qualitativo riferito alla didattica dell'italiano erogata presso istituzioni come gli IIC, deve pianificare e monitorare il fenomeno linguistico ad ogni fase del suo processo di fruizione, esplorando non solo i contenuti ma anche la forma dell'evento linguistico: ciò determina un piano di sviluppo e una struttura sociale delle azioni volte a stabilire che cosa, il perché, il quando, il dove, il come lavorare; si è d'accordo con Butera (1980) nel definire le pratiche di lavoro con una cooperazione socializzata nel contenuto e nella forma. In questo contesto, si darà spazio al valore e alle competenze dei partecipanti, in particolar modo dei docenti, strutturando di fatto dei percorsi di engagement, di identificazione, di costruzione dei saperi nella varietà delle sue forme che investono gli aspetti didattici ed extra didattici dei corsi.

All'interno di questa cornice, il docente si riappropria delle idee e del valore che conferisce al prodotto lingua grazie all'opportunità di una formazione continua, di riflessione e di controllo del proprio operare. Da questo punto di vista, la negoziabilità che il docente ha di richiedere all'organizzazione è strettamente connesse al grado di consapevolezza metodologica della lingua e della qualità che riesce a diversificare, sotto forma di cultura tecnica, a lezione. Si fa nuovamente strada l'importanza della formazione come fenomeno cruciale per la qualità dell'insegnamento. Esso abbraccia un percorso temporale a lungo temine utilizzando tutte le forme di fruizioni possibile. A facilitare oggigiorno l'aggiornamento glottodidattico vi è poi la tecnologia che ha ampliato i territori della conoscenza dando modo ai docenti di accedere a situazioni formative meno suscettibili della distanza. 
Analisi sul ruolo degli istituti italiani di cultura e ipotesi di progettazione di nuovi ambienti di apprendimento a rete per la formazione on line dei docenti Giuseppe Maugeri, Graziano Serragiotto

Le prospettiva di qualità e di miglioramento continuo dell'organizzazione si basano sull'esigenza di creare:

a) un gruppo di docenti che sono parte vitale, gestionale ed esecutiva dell'offerta linguistica. Essi veicolano la qualità didattica del prodotto attraverso un orientamento simile e forme metodologiche personalizzate sugli stili di apprendimento degli allievi;

b) una cultura di gruppo che si allinea ai processi di costruzione del sapere dell'organismo, aggiungendo o arricchendola con nuovi punti di vista e definizioni. In questo modo, vengono determinate continuamente nuove situazioni di crescita collettiva;

c) una comunità che, integrando la storia dell'organizzazione, diventa soggetto narrante di idee, di pratiche e di esperienze condivise (Schein, 1990);

d) da punti sopra indicati, la comunità dei docenti garantisce l'equilibrio dell'organizzazione didattica per la centralità che essi hanno mantenere la qualità dei corsi, per i processi decisionali in cui ognuno è coinvolto, per le responsabilità a cui assolvono, per il fatto di esprimere professionalità differenti, per i legami forti instaurati tra pari e col target, per gli interessi e le influenze che si ricevono e danno durante le ore lavorative, per la gestione del tempo e così via.

Nel modello culturale e qualitativo di un prodotto che visibilizza i fondamenti valoriali dell'organizzazione come gli IIC, il corpo dei docenti assume una forte rilevanza per il senso di appartenenza e un sentimento di partecipazione alla realtà organizzativa della didattica che consentirà di costituire una comunità di persone che insegnano e lavorano insieme, che condividono una cultura e una vision, degli obiettivi e delle finalità estese a tutti i processi di 
Analisi sul ruolo degli istituti italiani di cultura e ipotesi di progettazione di nuovi ambienti di apprendimento a rete per la formazione on line dei docenti

Giuseppe Maugeri, Graziano Serragiotto

apprendimento dello studente. $\mathrm{Si}$ viene pertanto a costituire una comunità pratica di docenti che trova nel senso e nell'azione locale dell'istituto un obiettivo e dei risultati. Questo riconoscimento nell'organizzazione presenta una forma di apprendimento continuo su scala locale. L'obiettivo che tale contributo si pone mira invece a estendere questo senso di appartenenza della comunità locale a una di livello di riconoscimento internazionale. A tal fine, si considererà analogo all'obiettivo citato avanzare l'ipotesi di un network di docenti nella convinzione che gli assunti che lo promuovono servano a progettare un processo di formazione glottodidattica che, estraneo ai fenomeni di breve corso, risulti più incisiva e idonea all'integrazione condivisa della storia degli IIC da parte dei loro rispettivi insegnanti.

\section{Un progetto a rete: la formazione docenti attraverso un network}

La qualità dell'insegnamento è frutto di un processo dinamico che contempla momenti di apprendimento collettivo e condiviso, spazi collaborativi e modalità sistemiche e progressive di riflessione sui contenuti e di problem solving che, combinandosi con l'esperienza degli individui, trovano valorizzazione nei percorsi formativi e di aggiornamento glottodidattico dei docenti.

Nel caso degli IIC, la formazione dei docenti è avvenuta finora attraverso l'articolazione di proposte e interventi esterni agli organismi, con un esborso di capitali che, in un quadro nazionale e politico di tagli, rappresentano un possibile deficit alle disponibilità di risorse dell'Istituto ${ }^{2}$. In tal senso, un'alternativa a incrementare i flussi di cassa investendo sul capitale umano, sarebbe quella di promuovere la cultura IIC favorendo l'aggiornamento glottodidattico della comunità dei docenti IIC che già apprendono e

\footnotetext{
${ }^{2} \mathrm{Si}$ considera interpretativo e illuminante il percorso di aggiornamento on line dei docenti svolto dall'IIC di Haifa, in Israele. Per il formarsi di comunità di pratica professionali, invece, si tenga in riferimento la comunità di pratica dei docenti di italiano LS/L2 consolidatasi a seguito e dopo l'erogazione del Master ITALS di II livello dell’Università Ca' Foscari di Venezia.
} 
Analisi sul ruolo degli istituti italiani di cultura e ipotesi di progettazione di nuovi ambienti di apprendimento a rete per la formazione on line dei docenti

Giuseppe Maugeri, Graziano Serragiotto

condividono in rete strumenti e contenuti, metodologie e materiali di tipo organizzativo e didattico. In questi termini e nei successivi paragrafi, si vogliono fornire gli assunti epistemologici su cui sviluppare l'ipotesi di un progetto formativo a rete dove implementare con l'uso delle nuove tecnologie livelli di intensità, di partecipazione e di coinvolgimento maggiore per una formazione glottodidattica on line rivolta alla crescita professionale del corpo docente IIC.

\subsection{Apprendere nell'era multimediale}

Perché sia tale e abbia dei risultati, il cambiamento necessita di un processo che consenta agli individui di adattarsi e ristrutturare attraverso l'utilizzo di nuove formule e canali, il senso della propria partecipazione e l'importanza di un apprendimento condiviso. Alla base dell'aggiornamento multimediale, vi è dunque una concezione apprenditiva interessata a cogliere il network personale dei legami, dei flussi di contenuti e di interessi condivisi che realizzano l'abbattimento delle resistenze al nuovo e promuovano una linea di apprendimento permanente mediata dalla tecnologia.

Si rende allora necessario progettare l'apprendimento affinché produca valori intrinseci al prodotto linguistico fruito, ovvero appartenenza, riconoscimento del valore aggiunto del fare e del sapere fare insieme, condivisione non solo di aspetti didattici ma di quei principi, criteri di comportamento e norme che regolano l'agire che ciascuno del gruppo assicurerà, in termini di qualità, alla sua attività di insegnamento. Partendo da questo presupposto, l'apprendimento diventa elemento prioritario per creare una comunità che abbia nell'ordine:

a) una dimensione di appartenenza e di identità;

b) una struttura a rete che poggi sui legami forti, sulla realizzazione di sinergie e di interdipendenze reciproche (Butera, 1999); 
Analisi sul ruolo degli istituti italiani di cultura e ipotesi di progettazione di nuovi ambienti di apprendimento a rete per la formazione on line dei docenti Giuseppe Maugeri, Graziano Serragiotto

c) una formula di ristrutturazione della conoscenza dal basso (bottom up).

Per tale ragione, la formazione necessita di essere progettata in ogni sua fase e continuamente, orientando la teoria verso una sua applicazione, con un grado elevato di partecipazione e di condivisione di dati che verranno diffusi in rete e in itinere dalla comunità professionale dei docenti. Dunque, nell'ambito della presentazione e della comprensione degli elementi essenziali di tali percorsi, gli aspetti significativi sono:

a) una progettualità che ha come tratto distintivo la natura multipla delle connessioni da sviluppare (Olson, 1979; Calvani, 2004): si tratta di concepire il movimento della comunità dei docenti con la stessa forma che si vuole dare e diffondere alla conoscenza, vale a dire la rete;

b) da questa architettura scaturisce un agire comunicativo coordinato da presupposti scientifici e di dinamica organizzativa mai episodici (Balboni, 2007);

c) lo sfruttamento delle potenzialità delle moderne tecnologie per raggiungere un ampio numero di destinatari legati da pratiche professionali attinenti l'insegnamento presso istituzioni come gli IIC;

d) la costituzione di percorsi che si diffondono ed evolvono parallelamente alla diffusione della tecnologia e dei moderni dispositivi utilizzabili per l'insegnamento e apprendimento delle lingue straniere (Ferrari, 2012);

e) se da un versante (punto d)la tecnologia ridisegna i percorsi formativi all'interno di un sistema, dall'altro le NT configura un diverso modo con cui il sistema entra in contatto con gli individui, incidendo sul modo di generare le relazioni interne a comunità professionali con interessi omogenei;

f) l'emergere di comunità di pratiche; nella concezione dell'apprendimento che si vuole definire, oltre a prendere in stretta 
Analisi sul ruolo degli istituti italiani di cultura e ipotesi di progettazione di nuovi ambienti di apprendimento a rete per la formazione on line dei docenti Giuseppe Maugeri, Graziano Serragiotto

considerazione gli studi di Wenger (1999) sulle comunità di pratica, si fa riferimento ai modelli di costruzione della conoscenza sorti in ambito aziendale e trasferibili al settore glottodidattico. Da questa premessa, le comunità di pratica sorte a seguito di questi percorsi collocano la loro sfera operativa all'interno di una medesima struttura a rete diffusa in più territori, così come sono distribuiti gli 89 IIC nel mondo;

g) la variazione della condizioni iniziali di partenza del sistema di apprendimento prefigura uno scenario che consente alla comunità, una volta formata e acquisiti gli strumenti concettuali ed esecutivi, di autogestirsi per semplificare ulteriori percorsi formativi condotti in scala più larga, per aree o microaree geografiche. In quest'ottica, la comunità di pratica diventa un modello di apprendimento in evoluzione, assumendo i tratti distintivi di una comunità che genera, negozia e condivide rispettivamente la conoscenza sugli argomenti e la competenza operativa sulla didattica dell'italiano a stranieri.

Quanto detto sopra, sottolinea l'importanza della dipendenza dallo stato iniziale di partenza, per cui l'implementazione di un percorso a rete di questo tipo richiede necessariamente le seguenti soluzioni:

a) una cambiamento nell'IIC della visione strategica di fare cultura, in sintonia con le esigenze del personale, del target, del mercato, dell'evoluzione tecnologica (Maugeri, 2013);

b) una struttura circolatoria e virtuale della conoscenza che abbia una valenza teorico-pratica (Coonan, 2002);

c) un coordinamento accuratamente esteso a ogni passaggio comunicativo, informativo con l'utente, nonché di strutturazione del processo di apprendimento; 
Analisi sul ruolo degli istituti italiani di cultura e ipotesi di progettazione di nuovi ambienti di apprendimento a rete per la formazione on line dei docenti Giuseppe Maugeri, Graziano Serragiotto

d) l'analisi dei luoghi virtuali aumentati da un punto di vista multisensoriale e cognitivo perché favoriscano l'espressione delle capacità di ciascuno dei partecipanti;

e) addestramento all'uso della tecnologia di modo che risulti essere funzionale allo scopo di accessibilità e circolazione e comprensibilità dei contenuti;

f) una attenta pianificazione delle attività orientate a elaborare i contenuti;

g) il monitoraggio della gestione dei corsi e dei contenuti si deve configurare come un servizio reso agli apprendenti con lo scopo di mantenere aperta la comunicazione, rendere disponibile le risorse, risolvere criticità relative agli scopi di apprendimento.

In tal modo, si forma una struttura a rete di attori e del loro sapere che va combinato, integrato, approfondito, arricchito e scambiato lungo l'asso temporale di costruzione della realtà. Alla base vi è quindi un modello socio costruttivo della conoscenza a cui si giunge grazie a un processo esperienziale e generativo nel quale l'individuo si posiziona sulla storia di altri individui (contesto globale) che radicano la loro riflessività e azione nell'ambito di contesti locali in cui ciascuno sviluppa un proprio universo culturale, linguistico e comunicativo di riferimento.

\subsection{Evoluzione tecnologica e fruirione multimediale dei contenuti di apprendimento}

Il focus che si terrà presente in questo paragrafo è relativo ai nuovi canali multimediali come strumenti di fruizione dei contenuti per l'apprendimento. Infatti, la tecnologica rappresenta un fenomeno costantemente in evoluzione, complesso e di tendenza. Proprietà pervasive che rendono funzionale e multiuso l'utilizzo della tecnologia sono:

a) l'ibridazione dei mezzi, ovvero la convergenza delle diverse applicazioni in una piattaforma specifica (Boaretto et. al, 2011); 
Analisi sul ruolo degli istituti italiani di cultura e ipotesi di progettazione di nuovi ambienti di apprendimento a rete per la formazione on line dei docenti Giuseppe Maugeri, Graziano Serragiotto

b) la fruizione simultanea dei canali in un unico strumento che si presta a convogliare le funzionalità dei dispositivi e a qualificarsi come mezzo potenziale di impiego per la ricerca di contenuti utili nella vita professionale e quotidiana;

c) il comportamento di consumo da parte degli individui che propende soprattutto per l'uso "sociale" dei moderni canali di comunicazione: questo fenomeno spiegherebbe il livello di interattività ricercato dall'utente per usufruire, in ogni istante e luogo, dei servizi di proprio interesse.

In quest'ottica, una strategia multicanale potrebbe facilitare e rendere operativi tutti i momenti, trasformando in modo attuale, mobile e peculiare il percorso di formazione on line grazie alla disponibilità e versatilità dei canali e dei contenuti da studiare e da realizzare. Si struttura così un sistema di offerta personalizzato che converge negli elevati bisogni del docente di costruire il proprio percorso professionale secondo una declinazione di forme di interazione sociale (Piaget, 1963; Bruner, 2003) che pongono l'individuo nelle condizioni di essere sempre connesso, aggregandosi ad altre comunità di pratica (Wenger, 1999).

\subsection{Proprietà e benefici degli ambienti di apprendimento on line}

La traiettoria di senso che trova riscontro nell'incontro con gli altri si dispiega all'interno di uno spazio organizzato che i nuovi sistemi rendono inscindibile dalla dimensione individuale e dalla stretta convivenza di rigore scientifico e creatività. Si tratta di un'area virtuale dove vengono costruiti scenari sempre diversi e in cui vengono inscritte le esperienze di crescita professionale delle persone. Gli ambienti di apprendimento virtuali risultano perciò essere funzionali all'incontro, allo spazio sociale dove la parola orienta all'azione, 
Analisi sul ruolo degli istituti italiani di cultura e ipotesi di progettazione di nuovi ambienti di apprendimento a rete per la formazione on line dei docenti Giuseppe Maugeri, Graziano Serragiotto

spinge ad analizzare, a riflettere su alternative possibili e ad agire. In tali spazi, la comunicazione diventa storia simbolica del passaggio dell'individuo all'interno della vita della comunità: è traccia di elevata competenza se si partecipa alla trasformazione dei saperi interiorizzando le manifestazioni più specifiche dell'intelligenza collettiva.

'In questo senso, l'elemento cruciale nell'apprendimento è che l'organismo è cosciente delle differenze e delle alternative e ha consapevolmente scelto una di queste alternative. La scelta può essere non di ricostruire un comportamento ma, piuttosto, di cambiare le proprie mappe cognitive o di comprensione". (Nostra traduzione da Friedlander, 1983, p. 194).

La funzionalità di un processo di apprendimento si misura sulla base della trasformazione che l'apprendente manifesta a seguito dell'avvenuta acquisizione. La potenzialità della conoscenza dunque giova alla comunità nel momento stesso in cui il corsista processa tale livello conoscitivo come utile per la crescita degli altri (Sims, et al. 1986) lungo un arco temporale permanente (la continuità dell'apprendimento come valore).

Entro questo scenario che dà luogo e forma alla collaborazione multimediale, a forme di lavoro cooperativo nonché alla condivisione di pratiche su scala globale, ogni membro della comunità risulta essere parte di questa trasformazione se:

a) concorre a aumentare le azioni/applicazioni della conoscenza all'interno di questa dimensione intermedia (fig. 3);

b) è in grado di ridisegnare i saperi attraverso nuovi canali, relazioni sociali nati sul web e pratiche didattiche innovative, cioè caratterizzate dall'utilizzo di nuove competenze e strumenti per la didattica della lingua italiana. 
Analisi sul ruolo degli istituti italiani di cultura e ipotesi di progettazione di nuovi ambienti di apprendimento a rete per la formazione on line dei docenti Giuseppe Maugeri, Graziano Serragiotto

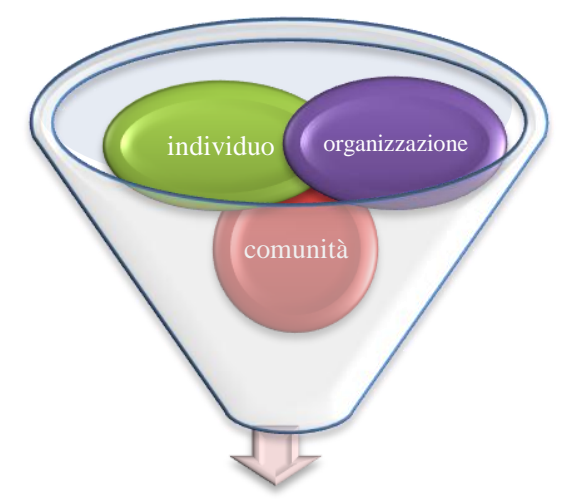

Ambiente di apprendimento in internet

Fig.3 Ambiente di apprendimento basato su internet. Nostra elaborazione.

Gli aspetti concettuali più significativi di questa tesi sono:

a) il costituirsi di luoghi privilegiati l'apprendimento glottodidattico entro un contesto organizzativo IIC che ha consentito mediante l'introduzione delle moderne tecnologie di far emergere una coscienza comunitaria del corpo docente;

b) una pianificazione delle attività strutturata e coordinata nei contenuti quanto nella forma;

c) l'utilizzo delle tecnologie rende sostenibile un modello formativo per cui la distanza fisica è alla base di una nuova modalità di aggiornamento di competenze di elevata competitività;

d) la tecnologia per la formazione rappresenta un vantaggio competitivo in quanto vengono abbattuti i costi di attività che prima risultavano efficaci solo se svolte fisicamente vicine;

e) i docenti non sono più considerati una risorsa piatta per gli IIC ma diventano un capitale intellettuale su cui investire per conferire ai corsi di lingua italiana un'ottica di qualità in funzione degli obiettivi di apprendimento degli studenti e del business dell'organizzazione (ovvero i principi del marketing didattico@), Maugeri, Serragiotto, 2013). 
Analisi sul ruolo degli istituti italiani di cultura e ipotesi di progettazione di nuovi ambienti di apprendimento a rete per la formazione on line dei docenti

Giuseppe Maugeri, Graziano Serragiotto

A corollario di questo paradigma, vi è l'ampio movimento che i destinatari assumono dalla definizione ed erogazione di un tale modello: i corsisti infatti, imposteranno un agire funzionale alla divisione del lavoro comune, allo sviluppo di una cultura IIC orientata al problem solving, all'utilizzo di modelli e tecniche che incrementano la qualità della loro azione didattica. Ciò darà la possibilità a ciascuno di svolgere un ruolo chiaro e definito di servizio per la comunità che gestisce in modo consapevole, organizzato e strutturato la cultura dell'organizzazione e il sapere. Verranno individuate e stabiliti stili comportamentali, vincoli e criteri temporali entro i quali l'intervento di ciascuno dovrà accompagnare il lavoro di riflessione degli altri; lo si farà in modo rilevante sia per il compito assegnato che per l'utilizzo di strumenti adeguati alle attività operative da svolgere in classe.

A conclusione, si possono distinguere due fasi riscontrabili nella formazione della comunità di pratica professionale dei docenti: la prima riguarda la condizione iniziale di formazione e di sviluppo della comunità attraverso dei percorsi di formazione. In questo caso, vi è l'esigenza di coordinare il processo, le forme di comunicazione e cooperative di lavoro. Successivamente, una volta che i legami risultino forti e gli individui sono soliti decidere insieme all'interno, si svilupperà un sistema auto poietico che, servendosi della tecnologia, elaborerà delle forme di adattamento alla conoscenza, integrandola con altri ambiti della ricerca (Celentin, 2007), sviluppandola ed estendendola a nuovi gruppi di docenti. Si riscontrano quindi diversi gradi di autonomia della comunità che vuole continuare a permanere in forma di apprendimento continuo (Senge, 1990), dialogica e simbolica di un partecipazione e interazione aperta alla conoscenza con un grado elevato di riflessività e di azione.

\subsection{Strategia metodologica e operativa per la formazione on line}


Analisi sul ruolo degli istituti italiani di cultura e ipotesi di progettazione di nuovi ambienti di apprendimento a rete per la formazione on line dei docenti Giuseppe Maugeri, Graziano Serragiotto

Vi è un ampio spettro di studi e di ricerche in merito al livello di acquisizione mediata dalla tecnologia (Caon, Serragiotto, 2012). Negli ambienti di apprendimento interni alla sfera di azione di programmi dedicati alla formazione dei docenti, la conoscenza ha un valore in parte critico la cui funzione consiste nel creare consapevolezza circa le modalità e gli indirizzi che tale metodologia glottodidattica è in grado di rivolgere e sperimentare con gli studenti.

Le mete glottodidattiche dei corsi sono dunque conseguite mediante diversi tipi di modalità di apprendimento, in modo particolare il "learning by doing (impararare facendo)" e "il learning from and by others (imparare da e attraverso gli altri)". Si tratta di approcci che vengono svolti lungo un percorso formativo e che presentano la caratteristica di essere integrabili all'interno di una medesima logica che si pone l'obiettivo di costruire in modo coerente un sapere indirizzato alle abilità e alle specializzazioni degli insegnanti. La conoscenza in tal modo acquisita diventa bagaglio esperienziale e distintivo che raggiunge ampiezza e profondità nell'ottenimento da parte del docente di un repertorio tecnico che gli consente di individuare i bisogni degli studenti e di attuare delle soluzioni sempre pertinenti e adeguate allo sviluppo delle competenze meta cognitiva e metadisciplinare dell'allievo.

La specificità dei due approcci sopraccitati consistono, oltre alle proprietà e dinamiche che li contraddistinguono e per la descrizione delle quali si rimanda ai contributi di Varisco (2002) Dolci e Celentin (2003) presenti in bibliografia, nel distribuire e consegnare alla community dei contenuti che vengono esplorati, discussi, rielaborati sulla base della interazione sociale e della propria esperienza conoscitiva tacita e professionale. In questo modo gli utenti, hanno modo di organizzare la struttura e veicolarla nell'ambiente sotto forma di analisi e riflessioni sui risultati conseguiti. 
Analisi sul ruolo degli istituti italiani di cultura e ipotesi di progettazione di nuovi ambienti di apprendimento a rete per la formazione on line dei docenti Giuseppe Maugeri, Graziano Serragiotto

In questo modo, si configura una linea del tempo di apprendimento scalabile che, a prescindere dalla articolazione e scansione tematica con cui avvengono e sono gestiti i contenuti, determina un livello di attività conoscitiva diversa il cui status è continuamente aggiornato grazie all'introduzione di nuovi stimoli e canali per la socializzazione con gli altri membri. Entro tale prospettiva, l'individuo aumenta il suo apprendimento al passo della sua interazione e collaborazione con la comunità. Ne consegue lo sviluppo e successivo rafforzamento della propria motivazione ad imparare, dell' identità e del senso di poli appartenenza che si amplificano in effetti sociali (scambio, condivisione).

Lungo la curva di tale crescita cognitiva e professionale, si individuano delle fasi aperte che combinandosi in e fra ogni soggetto produce senso per l'intelligibilità dell'esperienza che innova lo stile comunicativo e relazionale dei partecipanti e il senso della loro acquisizione, comportando il superamento del proprio equilibrio per tendere verso un bilanciamento diverso e nuovi traguardi conoscitivi.

L'approccio socio costruttivistico quindi integra al proprio interno le due modalità di costruzione del sapere. Ogni membro è chiamato a partecipare attivamente e a collaborare interagendo e lavorando su precisi compiti grazie ai quali accumula esperienza e perfeziona il suo sapere. Perché tale procedimento diventa sapere specialistico, allora la comunità deve esprimere un parere negozio nato da tutti sul lavoro effettivo svolto, appropriandosi del procedimento adoperato da ciascuno per realizzare e conseguire quel determinato obiettivo. In tal modo, ogni candidato spiega le diversi fasi di lavoro presentando le diverse soluzioni apportate, cosicché il processo di creazione è oggetto di analisi, le informazioni e gli strumenti vengono processati e le diverse tecniche una volta spiegate diventano patrimonio di tutti e depositate nel sapere accumulato dalla comunità. Il feedback 
Analisi sul ruolo degli istituti italiani di cultura e ipotesi di progettazione di nuovi ambienti di apprendimento a rete per la formazione on line dei docenti

Giuseppe Maugeri, Graziano Serragiotto

rappresenta un momento conoscitivo e non soltanto valutativo del compito e nella prospettiva indagata la presentazione del lavoro è inserita in un contesto sociale per cui le chiavi della performance con cui il lavoro è stato eseguito consente a ciascuno di entrare nel merito del lavoro degli altri, di appropriarsene se produce una logica di confronto continua.

Attraverso questa struttura, si genera uno scambio multi direzionale che arricchisce e giustifica $\mathrm{i}$ lavori nella misura in cui si raggiunge tutti i componenti della comunità in modo simmetrico. In tal senso, la diffusione della conoscenza è trasferita dalla divisione in gruppi che convergono collettivamente nell'intelligenza della comunità.

\subsection{Le mete formative dell'apprendere in rete}

Il processo di organizazione generato acquisisce una valenza educativa e didattica determinante se risponde a un percorso inclusivo, aperto e integrato fra i diversi attori: organizzazione- individuo- comunità. Inoltre il processo innalza la curva di valore di apprendimento se è allineato ai valori della cultura IIC (fig. 4), supportando così l'attuazione di un percorso di maturazione della coscienza critica dell'individuo (Menichelli, 2012) come mostra la fig. 4.

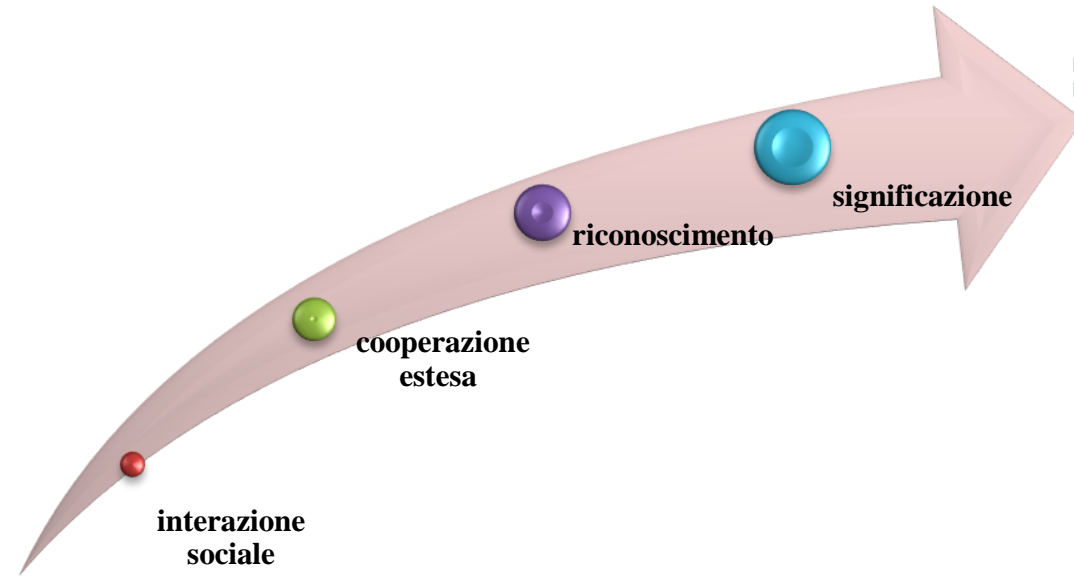

TRASFORMAZIONE

Fig. 4. Nostra elaborazione. 
Analisi sul ruolo degli istituti italiani di cultura e ipotesi di progettazione di nuovi ambienti di apprendimento a rete per la formazione on line dei docenti Giuseppe Maugeri, Graziano Serragiotto

Le meta formativa a cui giungere è un percorso che razionalizza e distribuisce i processi del sapere glottodidattico, consentendo poi di essere tradotti in competenze legate all'ambito operativo e didattico. Perché tale percorso risulti validante, è opportuno adeguare i percorsi alle esigenze specifiche (personalizzazione) del target. In parole concrete, verranno definite e indagate le aree ritenute essenziali in funzione dell'attività e degli interessi dei docenti.

Alla trasformazione si arriva mediante un vero e proprio flusso vitale di dati ed elementi che lo spazio virtuale costruito per l'aggiornamento, trova il modo di valorizzare sfruttando il bagaglio culturale dell'individuo e innescando su di esso nuovi elementi di riflessione e di miglioramento.

In questo modo, si configura una linea del tempo di apprendimento scalabile che, a prescindere dalla articolazione e scansione tematica con cui avvengono e sono gestiti i contenuti, determina un livello di attività conoscitiva in continua evoluzione, il cui status è continuamente aggiornato dal basso grazie:

a) alla responsabilità e alla disponibilità del docente/corsista di riorganizzare in maniera contestuale il suo apprendimento;

b) di valorizzare le sue nuove competenze attraverso una configurazione diversa della didattica, mettendo come parte del sistema di apprendimento i bisogni dello studente;

c) all'introduzione di nuovi stimoli e canali per la socializzazione e condivisione con gli altri membri della conoscenza e della creatività.

Entro tale prospettiva di azione collettiva, l'individuo non si limita alla ripetizione di didattismi e schemi obsoleti, ma egli stesso è responsabile principale della propria crescita; egli perciò aumenta il suo apprendimento al passo del suo aggiornamento professionale, del suo atteggiamento e disposizione a interagire, a mettersi in gioco e collaborare con la comunità professionale. Ne consegue lo sviluppo e successivo rafforzamento della propria motivazione ad imparare ad imparare, dell'identità e del senso di poli 
Analisi sul ruolo degli istituti italiani di cultura e ipotesi di progettazione di nuovi ambienti di apprendimento a rete per la formazione on line dei docenti

Giuseppe Maugeri, Graziano Serragiotto

appartenenza che si amplificano in effetti sociali (scambio, cooperazione, condivisione, diffusione) e in una differente configurazione all'interno della rete IIC.

\subsection{La formazione dei docenti on line come prodotto culturale}

L'utilizzo di un percorso formativo sovra territoriale risponde alla crescente domanda di formazione dei docenti e all'importanza che una modalità di aggiornamento come quella analizzata ha il più delle volte contribuito al successo di un modello organizzativo in grado di offrire/attivare dei percorsi metodologici alla didattica delle lingue straniere. Dagli effetti di questo orientamento, risulta quanto segue:

a) la cultura e specificatamente la formazione on line, rappresenta un'esperienza di business (Pine, Gilmore, 1998) che si fonda sulla esperienza di uso (Holbrook, Hirschman, 1982; Wolf, 1993), la quale è una caratteristica dei prodotti immateriali; ne consegue l'impostazione di una metodologia di studio (costruttivismo) che attribuisce senso e coerenza al processo esperienziale che la formazione attiva e amplia sotto forma di costruzione abilitante dei saperi;

b) su tale modello di business le organizzazioni hanno il compito di definire valore (Cesbrough, Rosenbloom, 2002) costruendo un sistema di offerte culturali i cui effetti sono la differenziazione, la rilevanza delle proposte attentamente calibrate sulla reale esigenza di uno specifico target e il presidio della rete di relazioni instaurate;

c) l'offerta culturale, messa nel mercato, mira a soddisfare le attese e le aspettative del cliente. Considerate l'immaterialità e la concentrazione nel web, essa si rende disponibile alla fruizione di tutti coloro che sono interessati a formarsi. La piattaforma di accesso è potenzialmente aperta e senza condizioni, a meno che non vi siano delle caratteristiche 
Analisi sul ruolo degli istituti italiani di cultura e ipotesi di progettazione di nuovi ambienti di apprendimento a rete per la formazione on line dei docenti

Giuseppe Maugeri, Graziano Serragiotto

curricolari e operative precise verso cui l'ente, per finalità e logiche istituzionali, propende assecondare;

d) elemento costitutivo e trasversale dell'offerta è la combinazione di conoscenza e creatività. Essa è la chiave per sostenere un modello culturale alla formazione innovativo e complesso. Inoltre, il suddetto modello diverge da quello tradizionale, frontale e in presenza, per una molteplicità di fattori tra i quali:

- la motivazione che spinge il docente a cimentarsi nei nuovi spazi digitali è strettamente interdipendente a una scala di bisogni che culminano nel riconoscimento del senso e del valore della sua cognizione;

- il canale di erogazione e di comunicazione;

- le modalità di comunicazione e la forma di espressione con cui essa si realizza (Bruner, 1990);

- gli ambienti di apprendimento e il ruolo giocato da tutti gli elementi compresi in tali spazi risultano essere progettabili (Dettori, Morselli, 2007) in favore di un apprendimento situato; da questo punto di vista, input e stimoli sensoriali (icone, spazi, colori, suoni) svolgono una parte attiva nel processo esperienziale di apprendimento, integrando la catena di valore che si snoda lungo i processi chiave secondo una logica situata, coordinata e di sistema;

- la varietà degli stili relazionale e cognitivi che emergono;

- l'intensità del coinvolgimento (Serragiotto, 2012);

- il livello di connessione fra i corsisti;

- una modalità di lavoro che premia la collaborazione fra i partecipanti (Trentin, 2001);

- la fruizione e l'analisi cooperativa dei materiali; 
Analisi sul ruolo degli istituti italiani di cultura e ipotesi di progettazione di nuovi ambienti di apprendimento a rete per la formazione on line dei docenti

Giuseppe Maugeri, Graziano Serragiotto

- la costruzione della conoscenza (Varisco, 2002), ovvero il paradigma che risolve la dicotomia fra aspettative e i reali problemi formulati dai docenti avviene secondo uno svolgimento programmato delle cause in cui è possibile trovare delle soluzioni comuni, ricche e articolate di significato;

- la produzione di soluzioni uniche, che non ignorano le dinamiche dell'interazione, si collocano nella prospettiva di senso in merito al settore in cui si intende operare;

- la possibilità di passaggio da azioni sociali e di problem solving virtuali a giornate dedicate a riflessioni collettive svolte di presenza. Quest'ultimo aspetto dimostra come la comunità professionale opera lungo la traccia della rete ma non ha bisogno di essa per mutare in coerenza le proprie caratteristiche professionali.

A conclusione, si considerano un valore aggiunto alle pratiche di studio i principi della cooperazione e della condivisione di una cultura che procedendo dall'organizzazione, si diffonde in maniera ricorsiva a ogni membro, strutturando le forme più evolute di apprendimento e la natura delle stesse competenze sociali in reti di relazioni ibride. Si viene pertanto a creare un'esperienza di studio autentica perché essa è in grado di rispondere alle necessità formative degli insegnanti sostenuta da una ambiente semi strutturato, da un universo comunicativo che si apre alle forme della connessione, degli ipertesti e dai molteplici aspetti delle identità degli individui.

\section{Valutazione complessiva del progetto}

L'idea di un network di docenti che partecipano alla co-costruzione di significati e di soluzione di apprendimento risulta essere appropriato alla forma a rete degli IIC, dunque alla coerenza col sistema che apprende nel corso del suo sviluppo. Inoltre, affiancare all'aggiornamento frontale una 
Analisi sul ruolo degli istituti italiani di cultura e ipotesi di progettazione di nuovi ambienti di apprendimento a rete per la formazione on line dei docenti

Giuseppe Maugeri, Graziano Serragiotto

possibile azione formativa mediata dall'utilizzo della tecnologia, costituisce senza dubbio una modalità di investimento a medio lungo termine e di valorizzazione del capitale umano. Parlando della metodologia del corso e dei percorsi formativi, pare significativa l'adozione di un approccio coerente con il sistema ossia di tipo costruttivistico, poiché tale tipologia dà vita a dei percorsi modulati sulle aree formative dei destinatari e, soprattutto, avvia un meccanismo di esplorazione del contenuto che coincide con il loro accrescimento nel momento in cui è condiviso e sono, di volta in volta, processati gli spunti di riflessione, impostate le esperienze, negoziati i significati. L'obiettivo è di approfondire le diverse esigenze didattiche e formulare nuove proposte favorevoli a creare una dialogo utile a dar forma alla conoscenza attraverso dei percorsi e dei programmi arricchenti per la loro attualità e per la loro aderenza ai bisogni della comunità dei docenti.

Giunti a questo punto, si ritiene utile sintetizzare le opportunità di pratica che un tale metodo potrebbe realizzare. I benefici infatti che si possono ottenere sono di:

a) integrare in un contesto globale le aspirazione di aggiornamento espresse a livello glocale da professionisti del settore;

b) rafforzare il contatto e la collaborazione in uno spazio che rappresenta il sistema;

c) porre attenzione alla tecnologia per l'e-learning in modo da consolidare una modalità espressiva di aggiornamento legata allo sviluppo e alle potenzialità della rete;

d) giungere a una consapevolezza collettiva in riferimento a un metodo di lavoro orientato alla ricerca di soluzioni;

e) mantenere un equilibrio tra il valore creato dalla formazione e l'identità comune con cui l'IIC investa e rappresenta i docenti. Secondo questa visione strategica, gli insegnanti aumentano il senso di appartenenza 
Analisi sul ruolo degli istituti italiani di cultura e ipotesi di progettazione di nuovi ambienti di apprendimento a rete per la formazione on line dei docenti Giuseppe Maugeri, Graziano Serragiotto

all'istituzione tanto da diventare co-artefici dell'azione di promozione della lingua italiana.

\section{Considerazioni finali}

Con questo articolo si è voluto analizzare il vigente sistema di promozione linguistica e culturale italiano, considerando quei principi che, ispiratori del Sistema, rappresentano una caso illuminato della nuova cultura dell'apprendimento. Indirizzata al soggetto apprendente, i nuovi contesti imprenditoriali della cultura generano delle risposte innovative, mettendo nelle condizioni in chi è motivato a migliorare le proprie competenze di docente, di integrarsi nella comunità di riferimento glottodidattico reperibile laddove vi è connessione. In questa direzione, si colloca la proposta della formazione dei docenti IIC attraverso l'uso della tecnologia. Nulla di nuovo nel panorama dell'educazione linguistica se questo non riguardasse gli Istituti italiani di cultura nell'avvicinare la categoria dei docenti di cui dispone, allo scopo di innovare i corsi di italiani erogati e rendere maggiormente più immediati all'utilizzo i mezzi informatici come strumenti per qualificare, all'insegna dell'innovazione, le attività didattiche. Da questa proposta di ammodernamento inevitabile peraltro in uno spazio di apprendimento globale, comincia un movimento della tecnologia verso il fattore umano e, nello stesso tempo, dell'uomo verso l'innovazione tecnologica: essa viene umanizzata sicché diventa una modalità espressiva di formazione e di contaminazione del sapere. In quest'ottica, la rete diviene centrale di un mondo nuovo che programma e racconta le persone, ciò a cui essi aderiscono, il modo in cui i soggetti apprendenti partecipano e costruiscono il proprio bagaglio cognitivo ed esperienziale di ricchezza. In questi termini vanno interpretati i risultati che si attendono dalla comunità professionale dei docenti 
Analisi sul ruolo degli istituti italiani di cultura e ipotesi di progettazione di nuovi ambienti di apprendimento a rete per la formazione on line dei docenti Giuseppe Maugeri, Graziano Serragiotto

IIC: posizionata all'interno della glottodidattica italiana e internazionale, percorsa da una tensione ideale di miglioramento, essa si impegna a sviluppare e co-costruire dei processi di crescita cognitiva e di competenza metodologica, orientando il processo di insegnamento e apprendimento della lingua verso comportamenti didattici positivi e un sapere linguistico specializzato.

\section{BIBLIOGRAFIA}

ALVESSON, M. Prospettive culturali per l'organizzazione. Milano: Guerini e Associati, 1996.

ANDERSON, C. La coda lunga. Da un mercato di massa a una massa di mercati. Torino: Codice, 2010.

ARNOLD, P. et al. Narrative: Designing for context in virtual settings. In: FIGUEREIDO, A.D.; AFONSO, A.P. Managing Learning in Virtual 
Setting: the role of context. Hershey PA, Information Science Publishing, 2006. pp. 197-218.

BAKOS, Y; BRYNJOLFSSON, E. Organizational Partnerships and the Virtual Corporation. In Information Technology and Industrial Competitiveness: How Information Technology Shapes Competition. Boston, 1997. pp. 1-17.

BALBONI, P.E. Elementi di Glottodidattica. Brescia: La Scuola, 1985. (a cura di). Educazione letteraria e nuove tecnologie, Torino: UTET, 2004.

. Italiano lingua materna. Fondamenti di didattica. Torino: UTET, 2006.

- Qualità della politica, qualità dell'insegnamento. In Studi di Glottodidattica. 2007. Vol. 1, n. 3, pp. 1-7.

Storia dell'educazione linguistica in Italia. Dalla Legge Casati alla Riforma Gelmini. Torino: UTET, 2009.

Conoscenza, verità, etica nell'educazione linguistica. Perugia: Guerra, 2011.

BALBONI, P. E; MARGIOTTAA, U. (a cura di). Formare on line i docenti di lingue e italiano L2. Torino: UTET, 2008. 
BALBONI, P. E.; CAON, F. Abilità strategiche di comunicazione per il funzionario degli Esteri: dalla competenza linguistica alla competenza interculturale. In PETRI, F.; LOBASSO, F. Diplomathìa: l'arte di imparare due volte. Messaggi dal G8. Catanzaro: Rubbettino, 2010. pp. 27-42.

BANZATO, M. Apprendere in rete. Modelli e strumenti per l'E-learning. Torino: UTET, 2002

Modelli di e-learning. In TD Tecnologie Didattiche, 2005. Vol. 36, pp. 60-71.

BANZATO, M.; CORCIONE, D. Tecnologie didattiche. Modelli e ambiti applicativi delle ICT nella scuola. Bologna: CLUEB, 2007

BANZATO, M.; CORCIONE, D.; GUARDIGLI, G. Il tutor on line. Un quadro di riferimento per la certificazione delle competenze e della qualità. Bologna: CLUEB, 2007.

BETTETINI, G. La simulazione visiva. Inganno, finzione, poesia. Milano: Bompiani, 1991.

BLOOM, B. S. Tassonomia degli obiettivi educativi. Teramo: GiuntiLisciani, 1984

BJORKRGREN, D. The Culture Business. London: Routledge, 1996.

BOARETTO et al. Marketing reloaded. Milano: IlSole24Ore, 2011. 
BOLLO, A. I1 Marketing della cultura. Roma: Carocci, 2012.

BORELLO, E.; LUISE, M.C. L'offerta linguistica in Italia. Milano: UTET, 2007.

BRUNER, J. Acts of meaning. Cambridge, MA: Harvard University Press, 1990.

BRUNER, J. Making stories: law, literature, life. Cambridge, MA: Harvard University Press, 2003.

BUTERA, F., L'orologio e 1'organismo. Milano: Franco Angeli, 1984.

BUTERA, F. Il castello e la rete. Milano: Franco Angeli, 1990.

BUTERA, F., 1999, Il libro Verde della Pubblica Istruqione, Milano, Franco Angeli.

CALVANI, A. Educazione, comunicazione e nuovi media. Torino: UTET, 2004.

CALVANI, A.; ROTTA, M. Comunicazione e apprendimento in Internet. Didattica costruttivista in rete. Trento: Erikson, 1999.

CAON, F.; MARASCHIO, N. (a cura di). Le radici e le ali. L'italiano e il suo insegnamento a 150 anni dall'Unità d'Italia. Torino: UTET, 2011. 
CAON, F.; SERRAgIOTTO, G. Didattica e nuove tecnologie. Torino: UTET, 2012.

CARÙ, A.; COVA, B. The Impact of Service Elements on the Artistic Experience: The Case of Classical Music Concerts. In International Journal of Arts Management, 2005. 7, n. 2, pp. 39-54.

CELENTIN, P. Comunicare e far comunicare in internet. Venezia: Cafoscarina, 2007.

CHESBROUGH, H.W.; ROSENBLOOM, R.S. The role of the business model in capturing value from innovation: Evidence from Xerox Corporation's technology spin-off companies. In Industrial and Corporate Change, 2002. 11, n. 3., pp. 529-555.

CIMATTI, F. Fondamenti naturali della comunicazione. In GENSINI, S., (a cura di). Manuale della comunicazione. Carocci: Roma, 1999. pp. 53-88.

COLBERT, F. Il marketing delle arti e della cultura. Milano: ETAS, 2009.

COONAN, M. C. La lingua straniera veicolare. Torino: UTET, 2002

DE FELICE, L. Marketing conversazionale. Milano: Gruppo 24 ore, 2010.

DETTORI, G.; MORSELLI, F. Creare contesti di apprendimento mediante un'attività narrativa. In Tecnologie didattiche, 2007. 42, n. 3, pp. 25-31.

DI BARI, V., (a cura di). Web 2.0. Milano: IlSole24Ore, 2007. 
DISTRATIS, M. et. al. La dimensione fisica dell'innovazione. Milano: Franco Angeli, 2011.

DOLCI, R.; CELENTIN, P. La formazione di base del docente di italiano per stranieri. Roma: Bonacci, 2003.

DOLCI, R. Un impianto costruttivistico per la formazione on line. In BALBONI, P. E; MARGIOTTA, U. (a cura di). Formare on line i docenti di lingue e italiano L2. Torino: UTET, 2008. pp. 82-99.

DURKHEIM, E. Le regole del metodo sociologico. Roma: Editori Riuniti, 2004.

FABRIS, G. Il nuovo consumatore: verso il postmoderno. Milano: Franco Angeli, 2003.

FERRARI, S. Le tecnologie digitali per l'educazione linguistica. Milano: EDUCatt, 2012.

FRANCESCHINI, F. Quality Function Deployment. Milano: Ilsole24ore, 1998.

FRATERRIGO, V.; MAUGERI, G. Il marketing culturale per una politica linguistica di qualità, in Lettere Italiane. Seoul, 2009. 27, pp. 229- 252.

FRATTER, I. Tecnologie per l'insegnamento delle lingue. Roma: Carocci, 2004. 
FRIEDLANDER, F. Patterns of Individual and Organizational Learning. In SRIVASTAVA, S. The executive mind: New insights in managerial thought and action. San Francisco: Jossey Bass, 1983. pp. 192-220.

GIDDENS, A. Sociologia. Bologna: Il Mulino, 1991.

GIOVANARDI, C.; TRIFONE, G. L'italiano nel mondo. Roma: Carocci, 2012.

HOFSTEDE, G. The interaction between national and organizational value system. In Journal of Management Studies, 1985. n. 22, pp. 347-357.

HOFSTEDE, G. et al. Measuring organizational cultures: A qualitative and qualitative study across twenty cases. In Administrative Science Quarterly, 1990. n. 10, pp. 286-316.

HOLBROOK, M. B.; HIRSCHMAN E, C. The Experiential Aspects of Consumption: Consumer Fantasies, Feelings, and Fun. In Journal of Consumer Research, 1982. n. 9, pp. 132-140.

KOTLER, P. Il marketing secondo Kotler. Milano: Il sole 24 Ore, 1999.

LAVE, J.; WENGER, E. Situated learning: legitimate peripheral participation. Cambridge University Press, 1991.

LEVY, P. L’intelligenza collettiva. Milano: Feltrinelli, 1996. 
Analisi sul ruolo degli istituti italiani di cultura e ipotesi di progettazione di nuovi ambienti di apprendimento a rete per la formazione on line dei docenti Giuseppe Maugeri, Graziano Serragiotto

MARgiotTA, U. Pensare in rete. Bologna: CLUEB, 1997.

MAUGERI, G. Riflessione sui contesti didattici dell'italiano all'estero. In IN.IT [di prossima pubblicazione], 2013.

MAUGeri, G.; SERragiotTo, G. Principi di marketing didattico applicati all'insegnamento/apprendimento dell'italiano come lingua straniera [di prossima pubblicazione], 2013.

MELERO, C. Mezzi informatici per l'accessibilità glottodidattica: riferimenti teorici e proposte di applicazione. In DALOISO, M. (a cura di). Globes. Glottodidattica per i bisogni educativi speciali, numero monografico di Educazione Linguistica. Edizioni Ca' Foscari, 2012. pp. 65-78.

MENICHELLI, C. Media education e Formazione interculturale: una prospettiva comune? In ITALS, 2012. anno X, n. 29, pp.71-92.

MESSINA L. L'apprendimento come processo attivo e costruttivo. In Scuola Viva, 1989. n. 9, pp. 23-29.

MEZZADRI, M. Internet nella didattica dell'italiano: la frontiera del presente. Perugia: Guerra, 2001.

. La qualità nell'insegnamento delle lingue straniere. Perugia: Guerra, 2005.

MIDORO, V. (a cura di). E-learning. Apprendere insieme in rete. Ortona: Menabò, 2002. 
NICKERSON, S. R. et al. The teaching of thinking. New York: Lawrence Earlbaum Hillsdate, 1985.

NONAKA, I.; TAKEUSHI, H. The knowledge-creating company. New York: Oxford University Press, 1995.

OLSON, D. R. Linguaggi media e processi educativi. Torino: Loescher, 1979.

PAVAN, E. (a cura di). Il lettore di italiano all'estero. Roma: Bonacci, 2005.

PFEFFER, J.; SALANCIK, G. The External Control of Organizations. New York: Harper \& Row, 1978.

PIAGET, J. The psychology of intelligence. New York: Routledge, 1963.

OXFORD, R. Language Learning Strategies: what every teacher should know. New York: Newbury House, 1990.

OUCHI, W. G.; WILKINS, A. L. Organization Culture. In Annual Review Sociology, 1985. n. 11, pp. 457-483.

PFEFFER, J. Competitive advantage through people. Boston: Harvard Business Press, 1994. 
Analisi sul ruolo degli istituti italiani di cultura e ipotesi di progettazione di nuovi ambienti di apprendimento a rete per la formazione on line dei docenti Giuseppe Maugeri, Graziano Serragiotto

PINE, J. II; GILMORE, J.H. Welcome to the Experience Economy. In Harvard Business Review. Luglio-Agosto, 1998. pp. 97-105.

PISHGHADAM, R. A Quantitative Analysis of the Relationship between Emotional Intelligence and Foreign Language Learning. In Electronic Journal of Foreign Language Teaching, 2009. Vol. 6, n.1, pp. 31-41.

PORCELLI, G.; DOLCI, R. Multimedialità e insegnamenti linguistici. Modelli informatici per la scuola. Torino: UTET, 1999.

RICOEUR, P. Explanation and Understanding: On Some Remarkable Connections Among the Theory of the Text, Theory of Action, and Theory of History. In The Philosophy of Paul Ricoeur: An Anthology of His Work. Boston: Beacon Press, 1978. pp. 149-166.

SALVALAGGIO, M. L'organizzazione e struttura di master on line. In BALBONI, P.E.; MARGIOTTA, U. (a cura di). Formare on line i docenti di lingue e italiano L2. Torino: UTET, 2008. pp. 207-222.

SANTIPOLO, M. (a cura di). L'italiano. Contesti di insegnamento in Italia e all'estero. Torino: UTET, 2006.

SCHEIN, E. H. Cultura d'azienda e leadership. Una prospettiva dinamica. Milano: Guerini e Associati, 1990.

SENGE, P. The fifth Discipline. New York: Currency Dubleday, 1990. 
Analisi sul ruolo degli istituti italiani di cultura e ipotesi di progettazione di nuovi ambienti di apprendimento a rete per la formazione on line dei docenti Giuseppe Maugeri, Graziano Serragiotto

SERRAGIOTTO, G. (a cura di). Le lingue straniere nella scuola. Torino: UTET, 2004.

La valutazione nel CLIL: format e griglie. In COONAN, M. C. (a cura di). CLIL: un nuovo ambiente di apprendimento. Venezia: Cafoscarina, 2006. pp. 213-220.

Lingua e contenuti disciplinari e/o trasversali attraverso il cinema. In Educazione Linguistica, 2012. vol. 1, pp. 1-9.

SERRAGIOTTOT, G.; MAUGERI, G. Nuove coordinate per lo sviluppo degli Istituti italiani di cultura in ambito organizzativo e didattico. In ITALS, 2012. vol. n. X, 29, pp. 93-126.

SEVERINO, F. Economia e marketing per la cultura. Milano: Franco Angeli, 2012.

SIMS, H.P. et al. The Thinking Organization. San Francisco: Jossey-Bass Publications, 1986.

SOLIMA L. L’impresa culturale. Milano: Franco Angeli, 2004.

SULKUNEN, P. Society made visible on the cultural sociology of Pierre Burdeu. In Acta Sociologica, 1982. n. 25, pp. 103-116.

SUTHERLAND, L. Developing problem solving expertise: the impact of instruction in a question analysis strategy. In Learning and Instruction, 2002. n. 12, pp.155-187. 
TRENTIN, G. Dalla formazione a distanza all'apprendimento in rete. Milano: Franco Angeli, 2001.

VARISCO, B. M. Costruttivismo socio-culturale. Roma: Carocci, 2002.

WEICK, K. E. Organizzare. Torino: Isedi, 1993.

WEICK, K. E. Senso e significato nell'organizzazione. Alla ricerca delle ambiguità. Milano: Raffaello Cortina, 1997.

WENGER E. Communities of Practice. Learning as a Social System. In System Thinker, 1998. n. 9. Disponibile su http://www.co-il.com/coil/knowledge-garden/cop/lss.shtml.

Communities of Practice: Learning, Meaning and Identity. Cambridge University Press, 1999.

WILLIAMS, M.; BURDEN. R. L. Psychology for Language Teachers. Cambridge: Cambridge University Press, 1997. 